\title{
Consumer Health Information Diversity Project
}

\author{
Marg Muir and Catherine-Anne Miller
}

\begin{abstract}
Objectives - The Consumer Health Information Diversity Project is developing principles and protocols for the translation of multilingual resources and the implementation of strategies to engage the Chinese and South Asian communities so that the resources gathered or produced will meet their needs. Current multilingual Web sites will be evaluated, and the hospital's consumer health Web site will be enhanced, increasing navigability and accessibility. Methods - The health information needs of consumers are determined through focus groups. Once confirmed, a variety of strategies are used in response. Results - Activities are ongoing. Hard copy and PDF resources have been identified and navigability and accessibility have been addressed. Training is required to make these materials more available to the communities of interest. Discussion - Although not completed, a number of strategies have been initiated jointly by two hospital departments. It is hoped that encouraging community involvement in all phases of the project will increase its sustainability.
\end{abstract}

\section{Introduction}

Language is one of the greatest barriers to accessing health care [1]. In a community significantly more diverse than the provincial average, this can have a tremendous impact on the way health care and health information are delivered. Trillium Health Centre serves the city of Mississauga and west Toronto in Ontario, Canada. These two areas boast a high immigrant population; close to $47 \%$ of the population of Mississauga was born outside Canada, and approximately $45 \%$ speak a language other than English. Private sector funding was obtained in response to the recommendations of a multicultural needs assessment and feedback from the hospital interpreters and health care providers. The funding was given to address the health promotion, education, and outreach issues related to the awareness of and access to interpretation, health information, and disease prevention services for culturally diverse and low-literacy community members. This project is a partnership involving the hospital (Health Information and Wellness Centres and Diversity Services) and local community agencies.

Consumer health information is very difficult to find in a number of languages. Many Web sites contain varied amounts of health information, but none is comprehensive. It is often necessary to explore five or six sites to find the language required, and even then, you may not find the topic desired. The majority of the interfaces are in English. Referring a non-English-speaking client to these sites, therefore, is not an option. These same difficulties arise with hard copy resources. The odd newly translated resource shows up from time to time. To keep on top of the new arrivals, one

M. Muir ${ }^{1}$ and C.-A. Miller. Health Information \& Wellness, Trillium Health Centre, 100 Queensway West, Mississauga, ON L5B 1B8, Canada.

${ }^{1}$ Corresponding author (e-mail: mmuir@thc.on.ca). must constantly be checking. You never know what might be translated next!

To address these issues, we embarked on the Consumer Health Information Diversity Outreach Project. The project has been most informative, confirming some of our original beliefs but also providing many new insights. The objectives of this project are as follows:

(i) To develop principles and protocols for the development of multilanguage materials for Trillium Health Centre as a whole

(ii) To develop a strategy to reach, engage, and identify health information needs for multicultural groups in the community

(iii) To develop new multilingual resources and (or) gather existing health information and disease prevention and management resources to meet identified priority needs

(iv) To enhance navigability and accessibility of a consumer health Web site for multilingual individuals and families

(v) To increase community and health care providers' awareness and use of existing multilanguage resources and services

Based on our knowledge of the populations of interest, which are predominantly of Chinese and South Asian descent (Chinese, Mandarin, Hindi, Punjabi, and Urdu are the languages spoken), we started our search for information needs with focus groups.

\section{Focus groups}

Through anecdotal information and discussions with the hospital interpreters, it became clear that diverse communities in Mississauga and west Toronto needed health information in their respective languages. To obtain specific information about their health information needs, focus groups were held in Mandarin, Cantonese, Punjabi, Hindi, and Urdu. Our community partners offered their meeting space, providing an accessible and comfortable location for 
the participants. The agencies also recruited participants, with two groups preferring to meet on Saturday! The majority were preformed groups. The focus groups did not exceed 1.5 hours in length. Each was led by a facilitator and cofacilitator who both spoke the groups' respective languages. Questions were developed to provide structure but were rephrased based on input from the specific groups. We learned that many of the terms that we use freely in English couldn't be translated into different languages. For instance, the term "health information" is not easily conveyed to a Chinese audience. Generally speaking, we asked the following questions: (i) What does good health mean to you? (ii) Who do you trust to give reliable health information? (iii) How do you remember health information? and (iv) What health concerns or health topics are you most interested in?

The dynamics in each group differed, but the responses were similar. Not surprisingly, the question of good health meant different things to different people. To some it centred on religion and sound mental health. For others, it was about physical health, a healthy lifestyle, and independence. When asked whom they trusted to give them reliable health information, the top response in both communities was the family doctor - but only for a brief summary of the information, not for the detailed points. The sources trusted for more specific information included family members with medical knowledge, pamphlets, the Internet, health organizations, media, and books. When asked how they remember health information, the participants shared many examples such as repetition, listening to tapes, posting notes on the refrigerator, etc. We had hoped to get a clearer breakdown to provide us with some direction when deciding on the format for new materials. Since the focus groups, pamphlets have come up in conversation most often as the format of choice. Because a number of these resources are available only in PDF, this presents an obstacle for a large number of individuals with the interfaces presently available.

The health concerns and topics voiced by the participants were many, spanning a broad range of health issues. When participants were probed about the information lacking in their communities, the responses became more specific. They identified the need to know more regarding how to find a family doctor, provincial health coverage, infectious diseases, mental health concerns, and how to use existing health services (e.g., the local hospital). Throughout the focus groups, immediate concerns and suggestions were articulated. Areas of complete and continual frustrationthat were mentioned by the participants included emergency room wait times and rushed family doctors.

\section{Program implementation}

Armed with this rich data, we had to address these concerns. We wanted to start working on some aspects immediately to keep the communities engaged. Since many strategies were going to take longer to put into place, identifying booklets and pamphlets already available in the languages of interest seemed manageable at the beginning. Some pamphlets were found in Chinese; fewer emerged in Hindi, Punjabi, and Urdu. One team member immediately started organizing the Web sites, enhancing the navigability and accessibility of the consumer health Web site for multilingual families and individuals. This included developing language appropriate interfaces (http://www.trilliumhealthcentre.org/health/ search_health_information/Languagelinkpage.html). The Chinese interface is complete. Unfortunately, the South Asian interface has proven more difficult owing to the challenges in integrating South Asian fonts into the hospital Web site. Specific information sites have been gathered for all languages, realizing, of course, that this is an ongoing process (i.e., sites are added continually).

To maintain the integrity of the site, we assessed all documents against developed criteria as we collected them from the many different organizations and sites. These criteria included the following:

(1) English versions of the documents had to be available so that the information could be reviewed.

(2) Documents had to be published in 2001-present. Dateless documents were excluded.

(3) Sites focusing only or mostly on localized community resources were excluded because they were deemed useless for our population.

These criteria did limit the number of documents available in some languages.

Another component of the program, which we have recently initiated, is the arranging of information sessions with community groups. The sessions are taking longer to organize than originally anticipated because of the degree of input required by the community agencies. Although we continue to strive for the greatest level of community involvement in this process, we must acknowledge that it is time-consuming. Because the original responses during the focus groups varied, it did not feel appropriate to deliver a predesigned presentation to different groups from different cultural backgrounds. Also, some participants want a formal presentation; others prefer an informal one. We continue to work closely with the community agency staff to ensure that we address their clients' concerns. As we invest in this segment of the project, some questions have arisen as to whether we are addressing this particular need in the most effective way. Do we have the staff to continue providing resource-intensive information sessions? Would providing information to leaders in the community ensure that the information reaches those in need? Our hope is that involvement in these sessions will soon highlight some common threads that may lead to a predesigned presentation, developed hand-in-hand with our community partners. Potentially, this presentation could be given without our staff.

A much anticipated component of the program was the Multilanguage Health Information Helplines. This was a collaborative effort between the Health Information and Wellness Centres and Diversity Services in the hospital. It was felt that it would assist clients as they navigate the health care system and enable them to get answers to the concerns discussed in the focus groups. These helplines are available in Cantonese, Mandarin, Punjabi, Urdu, and Hindi. The telephone helpline staff help our multilingual communities find a family doctor, locate community support and referrals, answer health questions, and book an interpreter, if required. At present, the lines are primarily on voice mail, with a promise to call back within 1 week. The volume of calls will be evaluated on an ongoing basis to determine 
whether changes are required (i.e., increase interpreter time). We had a big project launch in conjunction with Canada's National Multiculturalism Day to get the word out, as this has been a challenge. The ethnic media were present, increasing our exposure. We are also identifying places where information is typically available for these communities, for instance, grocery stores and temples.

To address sustainability with respect to the attainment of resources, "train the trainer" sessions will be offered to community agency staff and volunteers. These sessions will include information on how to navigate the Web site, how to access multilingual information, and how to order various documents from reliable sources. These training sessions are essential to empower communities to obtain their own health information and maintain an adequate supply of information in their agencies. Ensuring that the agencies know about the PDF documents available for downloading may provide greater accessibility when compared to hard copy resources. As stated previously, the identification of appropriate resources for the Chinese and South Asian communities was completed, and this knowledge should be transferred into the community.

One of our other goals is to create new multilingual resources. We are still in the process of determining what our communities need in terms of new materials. The information sessions may provide insights as to where the gaps exist. The need for drug information has been mentioned, so we are trying to assess whether translating drug profiles would be a helpful next step. Community pharmacists are already involved in this process. In an effort to standardize the translation process and give clear guidance, a translation protocol is being developed. Some excellent protocols have already been produced and tested, so it is not necessary for us to reinvent the wheel. Using other models $[2,3,4,5]$, we are following a protocol template used by our organization that takes into consideration the following: pre-translation, effective strategies, translation, review, proofreading, and evaluation.

\section{Evaluation}

The Consumer Health Information Diversity Outreach Project is being evaluated from a process and outcome perspective. The process evaluation allows us to reflect on our progress and methods, as well as determine whether or not we are meeting our stated goals. It provides the insight required to improve the program on an ongoing basis [6]. Since we hope to increase collaboration and partnerships with community agencies, we must monitor the activities that favor or discourage these relationships. Identifying what facilitates and challenges our process is important for the replication of the project and any adjustments throughout. The process evaluation takes the shape of monitoring activities, community input and involvement, resources used, and identifying changes and shifts in the project with time. The process measures also include staff time, thereby providing information on the need for increasing staff availability through time.
Outcome evaluation determines whether the project attained the stated goals and objectives. It takes the shape of quantifiable data such as statistics on Web site usage, the successful creation of new products and resources, and qualitative evaluation from the community members. The helplines are being evaluated by (1) monitoring their usage and (2) conducting a phone questionnaire, which is done by a member of the team as soon as possible following the initial call. The questionnaire includes measures for usefulness, the ease of the service, and general satisfaction. One of our stated goals was to develop resources to meet identified priority needs. Outcome measures to determine whether we are responding to these priority needs will take the form of usage data and community members' assessment of the resources. "Train the trainer" sessions will be evaluated for content, client satisfaction, and overall effectiveness.

\section{Discussion}

The Consumer Health Information Diversity Outreach Project has many components. As we develop one section, more parts emerge. This is what has made this project so interesting and exciting. There is no doubt that, as this initiative evolves and more partnerships develop, there will be much more to discuss. It is our hope that the helpline usage will increase, perhaps leading to a similar service for other ethnic communities. This, in combination with the Chinese and South Asian communities' self-sufficiency in obtaining hard copy and electronic materials, will, in part, address the sustainability issue. The project itself is time-limited, but hopefully its components are not!

\section{References}

1. Bowen S. Language barriers in access to health care. Ottawa: Health Canada; 2001 Nov. Available from http://www.hcsc.gc.ca/hcs-sss/pubs/acces/2001-lang-acces/index_e.html.

2. Agic B. Culture counts: best practices in community education in mental health and addiction with ethnoracial/ethnocultural communities. Toronto: Centre for Addiction and Mental Health; 2004 Oct 21. Available from http://www.camh.net/education/Resources_ communities_organizations/culture_counts_jan05.pdf

3. Downing BT, Bogosla LH. Translation as an effective strategy for effective communication with patients and clients: a how-toguide. Fresno (Calif.): Hablamos Juntos, UCSF Fresno Center for Medical Education and Research; 2003 Apr. Available from http://www.hablamosjuntos.org/pdf_files/BruceDowningHowTo_Guide_FINAL.pdf.

4. Downing BT, Bogosla LH. Effective patient-provider communication across language barriers: a focus on methods of translation. Fresno, Calif.: Hablamos Juntos, UCSF Fresno Center for Medical Education and Research; 2003 Feb 25. Available from http://www.hablamosjuntos.org/word_docs/BRUCEHJ_ Translation_Final_Feb03.doc.

5. Burroughs CM, Wood FB. Measuring the difference: guide to planning and evaluating health information outreach. Bethesda (Md.): National Library of Medicine, Office of Health Information Programs Development; 2000 Sept. 